\title{
Robustness evaluation of disturbance observer-based active queue management supporting TCP flows
}

\author{
Hosho Chibana $^{1 a)}$, Masashi Tadokoro², Daisuke Murayama ${ }^{3}$, \\ Ken-Ichi Suzuki ${ }^{2}$, and Ryogo Kubo ${ }^{1}$ \\ ${ }^{1}$ Department of Electronics and Electrical Engineering, Keio University, \\ 3-14-1 Hiyoshi, Kohoku-ku, Yokohama-shi, Kanagawa 223-8522, Japan \\ ${ }^{2}$ NTT Access Network Service Systems Laboratories, NTT Corporation, \\ 1-1 Hikarinooka, Yokosuka-shi, Kanagawa 239-0847, Japan \\ ${ }^{3}$ NTT Network Innovation Laboratories, NTT Corporation, \\ 1-1 Hikarinooka, Yokosuka-shi, Kanagawa 239-0847, Japan \\ a)chibana.hosho@kbl.elec.keio.ac.jp
}

\begin{abstract}
Implementation of active queue management (AQM) at routers can prevent serious congestion of transmission control protocol (TCP) flows. We previously proposed a disturbance observer (DOB)-based AQM controller to cope with modeling errors of network systems. However, the robustness of the controller against parameter fluctuations, e.g., the number of TCP connections and round-trip time (RTT) delay between terminal devices, has not been investigated sufficiently. This letter evaluates the robustness of the DOB-based AQM controller when the number of TCP connections and the RTT delay vary. Simulation results show that the DOBbased proportional-derivative (PD) controller provides better robustness than a classical proportional-integral-derivative (PID) controller.
\end{abstract}

Keywords: active queue management, transmission control protocol, congestion control, feedback control, disturbance observer

Classification: Network

\section{References}

[1] A. Molina-Garcia, J. A. Fuentes, E. Gomez-Lazaro, A. Bonastre, J. C. Campelo, and J. J. Serrano, "Development and assessment of a wireless sensor and actuator network for heating and cooling loads," IEEE Trans. Smart Grid, vol. 3, no. 3, pp. 1192-1202, Sept. 2012. DOI:10.1109/TSG.2012.2187542

[2] S. Floyd and V. Jacobson, "Random early detection gateways for congestion avoidance," IEEE/ACM Trans. Netw., vol. 1, no. 4, pp. 397-413, Aug. 1993. DOI:10. $1109 / 90.251892$

[3] C. V. Hollot, V. Misra, D. Towsley, and W. Gong, "Analysis and design of controllers for AQM routers supporting TCP flows," IEEE Trans. Automat. Contr., vol. 47, no. 6, pp. 945-959, June 2002. DOI:10.1109/TAC.2002.1008360

[4] A. Haider, H. Sirisena, and K. Pawlikowski, "PID based congestion control algorithms for AQM routers supporting TCP/IP flows," IEICE Trans. Commun., 
vol. E87-B, no. 3, pp. 548-555, Mar. 2004.

[5] R. Kubo, J. Kani, and Y. Fujimoto, "Advanced Internet congestion control using a disturbance observer," Proc. IEEE Global Communications Conference (GLOBECOM 2008), pp. 1-5, Dec. 2008. DOI:10.1109/GLOCOM.2008.ECP.269

[6] R. Kubo, J. Kani, and Y. Fujimoto, "Congestion control in TCP/AQM networks using a disturbance observer," IEEJ Trans. Ind. Appl., vol. 129-D, no. 6, pp. 541547, June 2009. (in Japanese) DOI:10.1541/ieejias.129.541

[7] "The network simulator - ns-2," online, http://www.isi.edu/nsnam/ns/.

\section{Introduction}

These days, an increasing number of consumer devices are connected to the Internet, and this leads to smart sensor-actuator networks (SANETs) being built [1]. The transmission control protocol (TCP) is utilized for reliable communication among the sensors and actuators. However, massive TCP flows in the SANETs cause serious congestion in routers. Active queue management (AQM) techniques discard packets in bottleneck routers to avoid serious congestion of TCP flows. For example, random early detection (RED) [2] discards packets with a drop probability determined according to the average queue length. Hollot et al. [3] designed the AQM controller on the basis of control theory. In addition, classical proportional-integral-derivative (PID) algorithms have been applied to the AQM controller [4].

The authors proposed the AQM controller using a disturbance observer (DOB) to address modelling errors of network systems [5]. The proposed controller includes a nominal model of the TCP/AQM network, and compensates the modelling errors calculated by the DOB. The effects of additional UDP flows on the DOB-based AQM controller was also assessed in [6]. However, the previous research studies did not consider substantial parameter fluctuations, e.g., the number of TCP connections and round-trip time (RTT) delay between terminal devices. This letter evaluates the robustness of the proposed DOB-based proportional-derivative (PD) controller when the number of TCP connections and the RTT delay vary. Simulation results show the effectiveness of the proposed controller in the presence of substantial parameter fluctuations, which may occur in future SANETs.

\section{TCPIAQM network model}

A model of TCP/AQM networks is shown in Fig. 1. In Fig. 1, $p, q$, and $C$ denote the packet drop probability, queue length of the bottleneck router, and bottleneck link capacity, respectively. The AQM controller implemented in the bottleneck router adjusts the packet drop probability $p$ according to the queue length $q$. In lossbased TCP algorithms, the congestion controller implemented in each sender node decreases the TCP window size when a packet loss occurs. Therefore, the AQM controller adjusts the TCP window size indirectly. The purpose of the AQM is to maintain the queue length $q$ at a target queue length $q_{0}$ and suppress the oscillations of $q$ so that buffer overflow and global synchronization are not caused. 


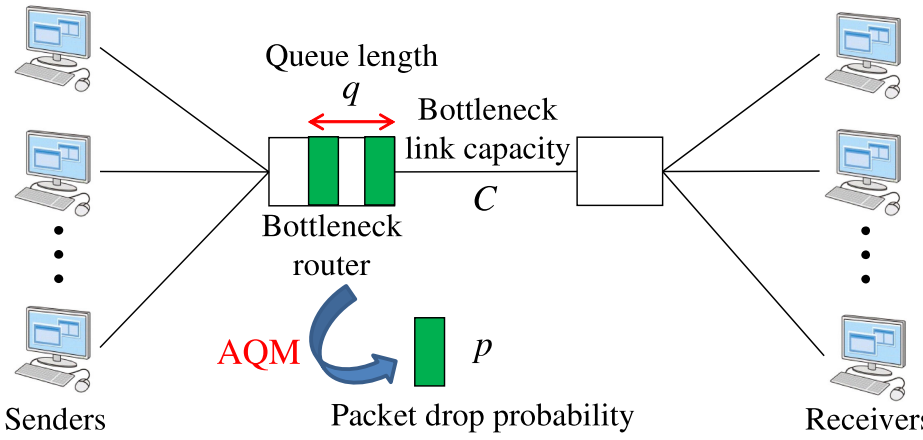

Fig. 1. TCP/AQM network.

The linearized system model of the TCP/AQM network, which was proposed in [3], is expressed as

$$
\begin{gathered}
\delta q=P(s) e^{-R s} \delta p, \\
P(s)=-\frac{\frac{C^{2}}{2 N}}{\left(s+\frac{2 N}{R^{2} C}\right)\left(s+\frac{1}{R}\right)},
\end{gathered}
$$

where $s, N$ and $R$ denote the Laplace operator, number of TCP connections, and RTT delay, respectively. In addition, $\delta q=q-q_{0}$ and $\delta p=p-p_{0}$ are the variation in the queue length and packet drop probability from the equilibrium points, i.e., $q_{0}$ and $p_{0}$.

In [5], we defined a simplified TCP/AQM network model $P_{n}(s)$ as

$$
\begin{aligned}
P_{n}(s) & =\frac{1}{M_{n} s^{2}}, \\
M_{n} & =-\frac{2 N_{n}}{C^{2}},
\end{aligned}
$$

where $M_{n}$ and $N_{n}$ are the nominal inertia of TCP/AQM dynamics and the nominal number of TCP connections, respectively.

\section{AQM controller design}

In this section, the PD controller, DOB, and total control system, including the proposed AQM controller for robust congestion control, are described.

\subsection{PD controller}

The PD controller calculates the referenced packet drop probability $\delta p^{r e f}$ from the queue length of the bottleneck router $q$. First, the referenced queue acceleration $\delta \ddot{q}^{\text {ref }}$ is calculated as

$$
\begin{aligned}
\delta \ddot{q}^{r e f} & =-C(s) \delta q \\
& =\left(K_{p}+K_{v} s\right)\left(q_{0}-q\right),
\end{aligned}
$$

where $K_{p}$ and $K_{v}$ are proportional and derivative gains. The referenced packet drop probability $\delta p^{r e f}$ is calculated as

$$
\delta p^{r e f}=M_{n} \delta \ddot{q}^{r e f} .
$$




\subsection{DOB}

The nominal inertia of TCP/AQM dynamics $M_{n}$ was utilized in designing the PD controller, and therefore, the modelling errors of TCP/AQM network are input to the control system as a disturbance $d_{d p}$ as

$$
d_{d p}=\left(P^{-1}(s)-P_{n}^{-1}(s)\right) \delta q,
$$

The DOB, including the artificial time delay $R_{n}$ and the nominal inertia $M_{n}$, estimates the disturbance $d_{d p}$ through a low pass filter as

$$
\begin{aligned}
\hat{d}_{d p} & =\frac{g_{\text {dis }}}{s+g_{d i s}}\left(\delta p_{a}^{r e f} e^{-R_{n} s}-M_{n} s^{2} \delta q\right) \\
& =\frac{g_{d i s}}{s+g_{d i s}} d_{d p},
\end{aligned}
$$

where $g_{\text {dis }}$ is a cut-off frequency of the DOB and $\delta p_{a}^{r e f}$ is the packet drop probability modified by the DOB. The DOB suppresses the disturbance $d_{d p}$ by modifying the referenced packet drop probability $\delta p^{r e f}$ as

$$
\delta p_{a}^{r e f}=\delta p^{r e f}+\hat{d}_{d p} .
$$

The modified packet drop probability $\delta p_{a}^{r e f}$ is set as an actual packet drop probability $\delta p$ in (1).

\subsection{Total control system}

The block diagram of the total control system is shown in Fig. 2. The system includes the PD controller, DOB, simplified TCP/AQM network model, and disturbance $d_{d p}$.

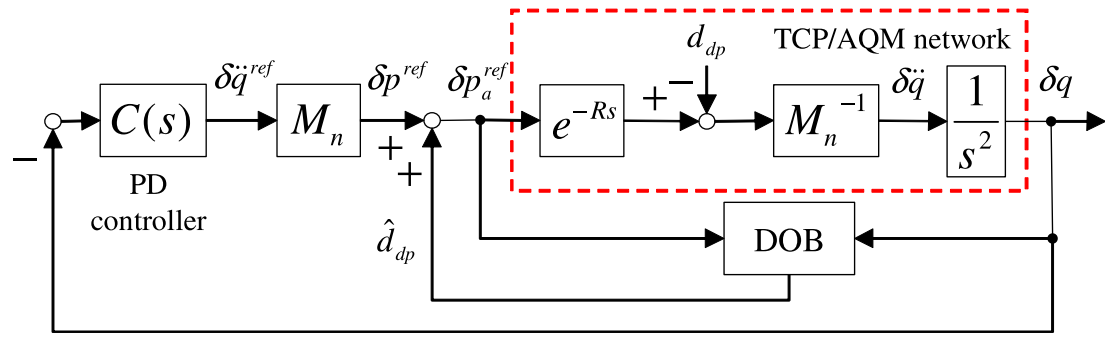

Fig. 2. Total control system.

\section{Robustness evaluation}

In this section, the evaluation of the robustness of the proposed DOB-based PD controller is described. Simulation results confirm the effectiveness of the proposed controller.

\subsection{Simulation setup}

The simulations were conducted using the network simulator ns-2 [7]. In the simulations, we compared the robustness of the proposed DOB-based PD controller and a classical PID controller without DOB as 


$$
\delta \ddot{q}^{r e f}=\left(K_{p}+\frac{K_{i}}{s}+K_{v} s\right)\left(q_{0}-q\right),
$$

where $K_{i}$ is an integral gain. The DOB can suppress the modelling errors as a disturbance, and this corresponds to the effect of the integral term in the classical feedback control theory. Therefore, the PID controller was chosen as a target for comparison in the evaluation. Other possible implementations of the feedback controller and other AQM controllers have been also proposed by many researchers. The comparison with the other controllers is considered a subject for future study.

In the simulations, the link capacity of access links, bottleneck link capacity $C$, and packet size were set to $10 \mathrm{Mbps}, 0.7 \mathrm{Mbps}$, and 1000 bytes, respectively. TCP Reno was utilized as an example of loss-based TCP algorithms. For the PID controller, the proportional gain $K_{p}$, integral gain $K_{i}$, and derivative gain $K_{v}$ were set to 900, 700, and 55, respectively. For the DOB-based PD controller, the proportional gain $K_{p}$, derivative gain $K_{v}$, and cutoff frequency of the DOB $g_{d i s}$ were set to 900,60 , and $50 \mathrm{rad} / \mathrm{s}$, respectively. The parameters were determined by the trial-and-error method in order to minimize the tracking error under the conditions $N=N_{n}$ and $R=R_{n}$.

We set the target queue length $q_{0}$ to 100 packets and the equilibrium point of the packet drop probability $p_{0}$ to zero. The average queue length and the standard deviation (SD) of queue length in the steady-state response were assessed. The simulations were performed while varying the number of TCP connections and RTT delay between senders and receivers. The nominal number of TCP connections $N_{n}$ was set to 50, and $N_{n}$ was utilized in designing the PD controller and DOB. However, the actual number of TCP connections $N$ varies dynamically. To confirm the robustness against the variation in the number of TCP connections, we set $N$ as $10,25,40,50$, or 100 . The artificial time delay $R_{n}$ was set to $200 \mathrm{~ms}$, and $R_{n}$ was utilized in designing the DOB. However, the actual RTT delay $R$ varies dynamically. To confirm the robustness against the variation in RTT delay, we set $R$ as 50, $100,200,400$, or $800 \mathrm{~ms}$.

\subsection{Simulation results}

The effects of the variation in the number of TCP connections, $N$, and the variation in RTT delay, $R$, are shown in Fig. 3(a) and Fig. 3(b), respectively. Not only the average queue length but also the $\mathrm{SD}$ of the queue length greatly affect the robustness of the TCP/AQM network system. A large SD indicates an oscillatory response of the queue length and may result in buffer overflow.

For the conventional PID and proposed DOB-based PD controllers, it was confirmed that a small $N$ increased the tracking error $q-q_{0}$ and the SD in Fig. 3(a), and a large $R$ increased the tracking error $q-q_{0}$ and the $\mathrm{SD}$ in Fig. 3(b). The proposed controller resulted in the same degree of tracking errors as did the conventional controller, and a great improvement in the SD of the queue length. The conventional controller could not handle substantial parameter fluctuations, although it compensated slight disturbances through the effect of the integral term. On the other hand, the proposed controller effectively suppressed the oscillations of the queue length. 


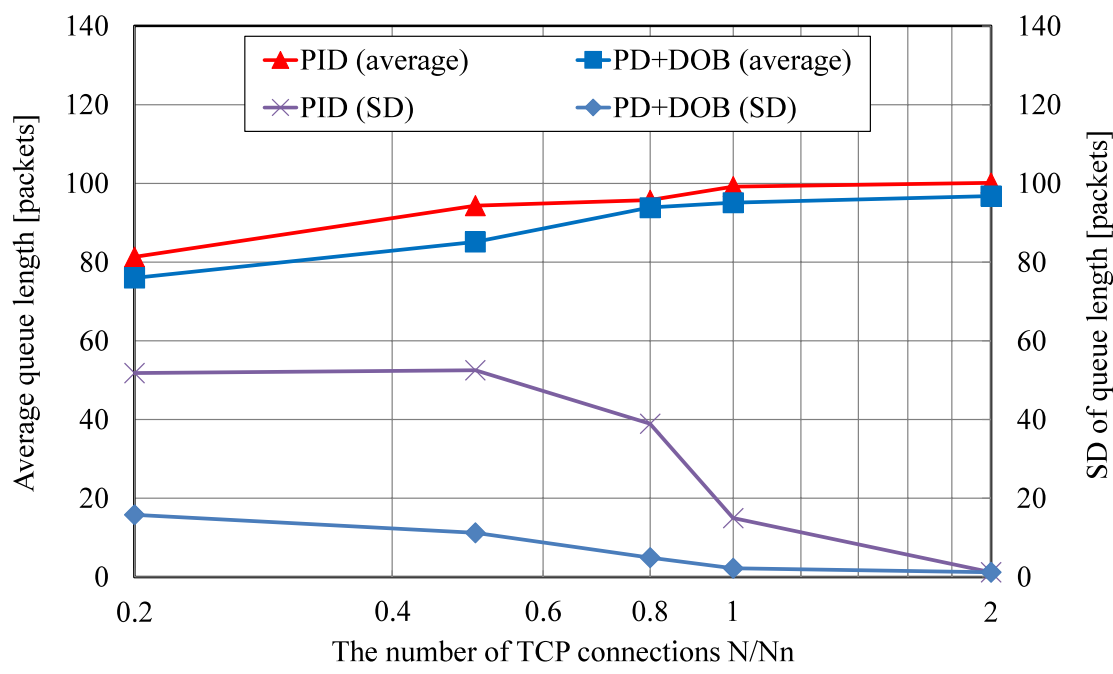

(a) Variation in the number of TCP connections

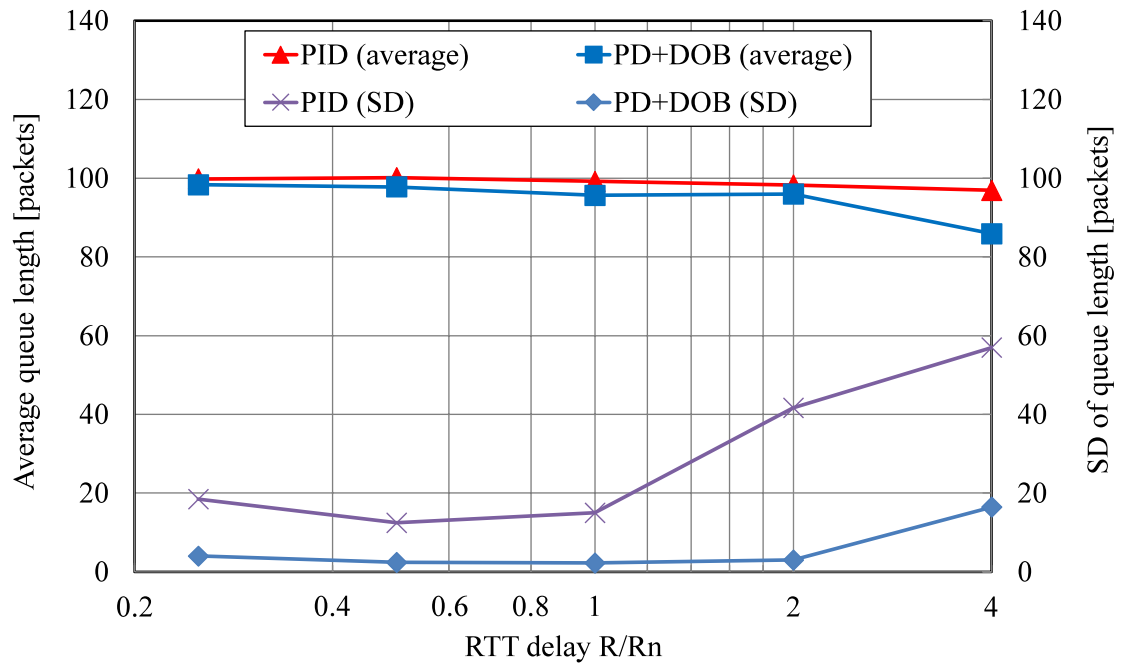

(b) Variation in RTT delay

Fig. 3. Simulation results.

\section{Conclusion}

In this letter, an evaluation of the robustness of the DOB-based PD controller for TCP/AQM networks was presented. The simulation results showed that the DOBbased PD controller provided better robustness against the variations in the number of TCP connections and RTT delay than did the classical PID controller. Our further study includes a comparison with other AQM controllers and simulations using other TCP versions. 